\title{
Brain Microstructure Reveals Early Abnormalities more than Two Years prior to Clinical Progression from Mild Cognitive Impairment to Alzheimer's Disease
}

\author{
Gwenaëlle Douaud, ${ }^{1}$ Ricarda A. L. Menke, ${ }^{1,8}$ Achim Gass, ${ }^{2}$ Andreas U. Monsch, ${ }^{3}$ Anil Rao, ${ }^{4}$ Brandon Whitcher, ${ }^{4,5}$ \\ Giovanna Zamboni, ${ }^{1,6}$ Paul M. Matthews, ${ }^{4,7}$ Marc Sollberger, ${ }^{2,3}$ and Stephen Smith ${ }^{1}$ \\ ${ }^{1}$ Functional MRI of the Brain (FMRIB) Centre, Nuffield Department of Clinical Neurosciences, University of Oxford, Oxford OX3 9DU, United Kingdom, \\ ${ }^{2}$ Departments of Neurology and Neuroradiology and ${ }^{3}$ Memory Clinic Department of Geriatrics, University Hospital Basel, 4031 Basel, Switzerland, \\ ${ }^{4}$ Imperial College London, London SW7 2AZ, United Kingdom, ${ }^{5}$ Mango Solutions, Chippenham SN14 0GB, United Kingdom, ${ }^{6}$ Oxford Project To Investigate \\ Memory and Ageing (OPTIMA), Nuffield Department of Clinical Medicine, University of Oxford, Oxford OX3 7BN, United Kingdom, ${ }^{7} \mathrm{GlaxoSmithKline,}$ \\ Clinical Imaging Centre, Hammersmith Hospital, London W12 0NN, United Kingdom, and ${ }^{8}$ Department of Psychiatry, Warneford Hospital, University of \\ Oxford, Oxford OX3 7JX, United Kingdom
}

Diffusion imaging is a promising marker of microstructural damage in neurodegenerative disorders, but interpretation of its relationship with underlying neuropathology can be complex. Here, we examined both volumetric and brain microstructure abnormalities in 13 amnestic patients with mild cognitive impairment (MCI), who progressed to probable Alzheimer's disease (AD) no earlier than 2 years after baseline scanning, in order to focus on early, and hence more sensitive, imaging markers. We compared them to 22 stable amnestic MCI patients with similar cognitive performance and episodic memory impairment but who did not show progression of symptoms for at least 3 years. Significant group differences were mainly found in the volume and microstructure of the left hippocampus, while white matter group differences were also found in the body of the fornix, left fimbria, and superior longitudinal fasciculus (SLF). Diffusion index abnormalities in the SLF were the sign of a subtle microstructural injury not detected by standard atrophy measures in the corresponding gray matter regions. The microstructural measure obtained in the left hippocampus using diffusion imaging showed the most substantial differences between the two groups and was the best single predictor of future progression to AD. An optimal prediction model (91\% accuracy, $85 \%$ sensitivity, $96 \%$ specificity) was obtained by combining MRI measures and CSF protein biomarkers. These results highlight the benefit of using the information of brain microstructural damage, in addition to traditional gray matter volume, to detect early, subtle abnormalities in MCI prior to clinical progression to probable AD and, in combination with CSF markers, to accurately predict such progression.

\section{Introduction}

A major goal for clinicians is to be able to detect which patients with mild cognitive impairment (MCI) are going to progress to Alzheimer's disease (AD) and develop clinical symptoms of dementia (Petersen, 2009). MCI has been recognized as an intermediate clinical stage between healthy aging and $\mathrm{AD}$, but not all $\mathrm{MCI}$ patients will progress to probable AD (Gauthier et al., 2006),

Received Sept. 18, 2012; revised Nov. 9, 2012; accepted Dec. 2, 2012.

Author contributions: G.D., R.A.L.M., A.G., A.U.M., G.Z., P.M.M., M.S., and S.S. designed research; G.D. and M.S. performed research; G.D., A.R., and B.W. contributed unpublished reagents/analytic tools; G.D. analyzed data; G.D., R.A.L.M., G.Z., P.M.M., and S.S. wrote the paper.

This work was supported by a UK Engineering and Physical Sciences Research Council grant (awarded to S.S.). These data were acquired as part of EAGLE, a longitudinal natural history study of Alzheimer's disease conducted at the University of Basel and funded by GlaxoSmithKline (GSK). G.Z. is supported by Oxford NIHR Biomedical Research Centre. We thank Dr. Nicola Filippini for providing help with statistical analyses.

P.M.M., B.W., and A.R. were, at the time of this study, full-time employees of GSK. The other authors declare no competing financial interests.

Correspondence should be addressed to Dr. Gwenaëlle Douaud, FMRIB Centre, University of Oxford, John Radcliffe Hospital, 0X3 9DU 0xford, UK. E-mail: douaud@fmrib.ox.ac.uk.

DOI:10.1523/JNEUROSCI.4437-12.2013

Copyright $\odot 2013$ the authors $\quad 0270-6474 / 13 / 332147-09 \$ 15.00 / 0$
(Ganguli, 2006, Maioli et al., 2007). Unsurprisingly therefore, numerous studies have investigated early signs of future progression to $\mathrm{AD}$ in MCI patients based on neuropsychological, CSF, and/or imaging data (Whitwell et al., 2008, Misra et al., 2009, Vemuri et al., 2009, Costafreda et al., 2011, Ewers et al., 2012).

However, despite some structural MRI studies focusing on the comparison of atrophy at baseline between the more homogeneous populations of stable amnestic MCI and progressing MCI, findings have so far been variable (Winblad et al., 2004, Maioli et al., 2007, Ferreira et al., 2011). Because small samples are the norm in the majority of these progression versus stable MCI studies, it is all the more imperative to have well defined criteria for the patient selection process, such as the time interval between baseline MRI and testing for progression to AD (Whitwell et al., 2008).

In addition, very few of these MRI-based progression versus stable MCI studies have investigated the brain tissue microstructural properties, partly because many have drawn on the Alzheimer's Disease Neuroimaging Initiative (ADNI) dataset in which diffusion imaging had not been acquired (Mueller et al., 2005). 
Table 1. Demographics of the two populations included in this imaging study

\begin{tabular}{llll}
\hline & $\begin{array}{l}s \mathrm{MCl} \\
(n=22)\end{array}$ & $\begin{array}{l}\mathrm{pMCl} \\
(n=13)\end{array}$ & $p$ value \\
\hline Age (years) & $69 \pm 9$ & $76 \pm 6$ & 0.02 \\
$\quad$ Age (years) for diffusion subjects only & $69 \pm 9$ & $76 \pm 6$ & 0.02 \\
Gender (F/M) & $11 / 11$ & $3 / 10$ & $0.23^{a}$ \\
$\quad$ Gender (F/M) for diffusion subjects only & $10 / 11$ & $3 / 9$ & $0.36^{a}$ \\
Period of follow-up/progression (months) & $47.5 \pm 4.5$ & $29.5 \pm 6.0$ & $<10^{-7}$ \\
$\quad$ Range (months) & $41-58$ & $25-41$ & \\
Disease duration (years) & $5.0 \pm 4.5$ & $3.5 \pm 2.0$ & 0.23 \\
APOE: Increased risk ( $\in 3 / \epsilon 4+\epsilon 4 / \epsilon 4)$ & $46 \%$ & $46 \%$ & - \\
$\quad$ No increased risk & $54 \%$ & $54 \%$ & - \\
Education & $13.5 \pm 3.5$ & $14.0 \pm 4.5$ & 0.72 \\
CSF markers (pg/ml): & & & \\
$\quad$ Tau & $320 \pm 100$ & $455 \pm 266$ & 0.10 \\
$\quad$ Phosphorylated tau & $54 \pm 13$ & $65 \pm 30$ & 0.23 \\
$\quad \beta$-Amyloid (A $\left.\beta_{42}\right)$ & $714 \pm 256$ & $513 \pm 221$ & 0.02 \\
\hline${ }^{a}$ Yates $\chi^{2}$ test. & & &
\end{tabular}

Of these few diffusion imaging studies, none found significant difference when directly comparing the two MCI populations (Kantarci et al., 2005, Fellgiebel et al., 2006, Haller et al., 2010, Oishi et al., 2011). One clue might reside in the complexity of the interpretation of the underlying neuropathology in white matter tracts, which requires the use of sensitive diffusion index markers and image processing (Douaud et al., 2011).

In this study, we compare two groups of amnestic MCI patients showing similar cognitive performance, episodic memory impairment, and disease duration and matched in proportion of apolipoprotein E (APOE) $\varepsilon 4$ carriers (Farrer et al., 1997, Trachtenberg et al., 2012). We chose to focus on amnestic MCI patients who progressed to probable AD no earlier than 2 years after their baseline scan ("pMCI"), in order to increase the predictive value of the imaging biomarkers evaluated here in a truly preclinical phase of the disease. We contrasted this group with amnestic MCI patients who were clinically stable (i.e., did not develop $\mathrm{AD}$ ) for at least 3 years following their first evaluation ("sMCI"). In these two pMCI and sMCI populations, we investigated without any spatial constraint on our analyses: (1) wholebrain gray matter volumetry using an optimized voxel-based morphometry (VBM) approach (Douaud et al., 2007); (2) gray matter microstructure using a voxel-based approach; and (3) white matter microstructure using the tract-based spatial statistics (TBSS) approach (Smith et al., 2006). We further tested the potential for integrated MRI-derived and CSF protein measures to discriminate amnestic MCI patients showing otherwise comparable neuropsychological performance.

\section{Materials and Methods}

\section{Participants}

This imaging study was part of the EAGLE (Early Alzheimer's disease Genetics - a Longitudinal Evaluation) study and was approved by the Ethics Committee of Both Basel (Switzerland). All subjects gave written informed consent.

In total, $64 \mathrm{MCI}$ patients of either sex were included in this study. CSF was collected in polypropylene tubes, centrifuged at $3000 \mathrm{rpm}$, divided into aliquots, and stored at $-80^{\circ} \mathrm{C}$ within $1 \mathrm{~h}$. The concentrations (in $\mathrm{pg} / \mathrm{ml})$ of total tau ( $\mathrm{t}$-tau), phosphorylated tau $181\left(\mathrm{p}-\mathrm{tau}_{181}\right)$, and $\beta$-amyloid 1-42 (A $\left.\beta_{1-42}\right)$ were measured according to the manufacturer's specifications by an experienced laboratory technician using a commercially available sandwich ELISA kit (Innogenetics) (Table 1). CSF samples were run in duplicates to detect variability and determine accurate concentrations. For all subjects, comprehensive neuropsychological data were also collected (Table 2). MCI subjects were diagnosed according to the criteria by Winblad et al. (2004) [mini-mental state examination
Table 2. Neuropsychological scores in the amnestic stable sMCl and progressing pMCl groups

\begin{tabular}{lccc}
\hline & \multicolumn{1}{l}{$\begin{array}{l}\text { sMCl } \\
(n=22)\end{array}$} & $\begin{array}{l}\text { pMCl } \\
(n=13)\end{array}$ & $p$ value \\
\hline MMSE & $28.5 \pm 1.5$ & $28.0 \pm 1.5$ & 0.15 \\
Boston Naming Test & $13.5 \pm 1.0$ & $13.5 \pm 1.5$ & 0.70 \\
Stroop (interference-color) & $20 \pm 7$ & $22 \pm 10$ & 0.62 \\
Digit span forward & $6.5 \pm 1.5$ & $7.0 \pm 2.0$ & 0.50 \\
Digit span backward & $5.0 \pm 1.5$ & $6.0 \pm 1.0$ & $0.02^{a}$ \\
Trail Making Test A & $47 \pm 20$ & $48 \pm 14$ & 0.91 \\
Trail Making Test B & $133 \pm 62$ & $131 \pm 51$ & 0.89 \\
Five-Point Test & $21 \pm 8$ & $19 \pm 7$ & 0.50 \\
Praxis: & & & \\
$\quad$ Pantomime & $9.5 \pm 1.0$ & $9.5 \pm 0.5$ & 0.35 \\
$\quad$ Sensory apraxia & $10.0 \pm 0.5$ & $10.0 \pm 0.5$ & 0.91 \\
$\quad$ Imitation & $12.0 \pm 0.5$ & $11.5 \pm 0.5$ & 0.07 \\
$\quad$ Bucco-facial & $6.0 \pm 0.0$ & $6.0 \pm 0.5$ & 0.34 \\
Verbal fluency (letters) & $12.5 \pm 4.5$ & $12.0 \pm 4.5$ & 0.70 \\
Verbal fluency (category) & $22.0 \pm 5.5$ & $18.0 \pm 5.0$ & 0.04 \\
Verbal delayed recall (normalized) & $-1.6 \pm 0.9$ & $-1.9 \pm 1.0$ & 0.38 \\
\hline
\end{tabular}

${ }^{a}$ The $\mathrm{pMCl}$ group performed better at this test.

(MMSE) $\geq 24 / 30$, global CDR (clinical dementia rating) score $\leq 0.5$, see Supplementary Information of Douaud et al., 2011 for more details]. All participants continued to be clinically monitored every 12 months following their first visit. Of the $64 \mathrm{MCI}$ patients, it appeared that 7 patients had displayed cognitive deficits due to other neurological reasons (temporal epilepsy, semantic dementia, dementia with Lewy bodies, hydrocephalus, alcohol-related, Parkinson's disease) and were therefore excluded from this study, as well as one imaging dataset being of too poor quality. Among the 56 remaining MCI patients, we created three groups:

-pMCI: 13 amnestic patients, who developed clinical symptoms consistent with a diagnosis of probable $\mathrm{AD}$ at least 2 years after their first visit, were selected to identify potential brain differences as early as possible (on average, 2.5 years; range, 25-41 months).

-sMCI: To ensure that the group of MCI patients remained stable for a longer period of time than the pMCI group, we selected the 22 amnestic MCI patients who remained stable for at least 3 years following their initial visit (on average, 4 years; range, 41-58 months) (Table 1). The longest period of clinical progression to $\mathrm{AD}$ for the $\mathrm{pMCI}$ group was the shortest period of confirmed clinical stability for the sMCI group (41 months).

- otherMCI: The remaining $21 \mathrm{MCI}$ patients who did not correspond to our "eligibility" criteria for the pMCI or sMCI groups were used for classification purposes in an additional analysis (see section below, Additional analysis: MRI and CSF classification of pMCI versus sMCI).

The pMCI or sMCI patients did not significantly differ in terms of disease duration or neuropsychological performance and, most particularly, they demonstrated comparable MMSE, episodic memory impairment, and duration of symptoms at the point of scanning (Table 2). The two pMCI and sMCI groups were also matched for the proportion of APOE $\varepsilon 4$ carriers with an increased risk of developing AD (Farrer et al., 1997, Trachtenberg et al., 2012) (Table 1). However, it has to be noted that, as a result of prioritizing the inclusion of all MCI patients who would meet our eligibility criteria of (1) a long period of progression to probable $\mathrm{AD}$ for the pMCI group $(n=13)$, thus truly focusing on the preclinical phase of an underlying process leading to $\mathrm{AD}$, and (2) a longer period of clinical stability for the sMCI group $(n=22)$, the groups differed in age ( 7 years). To make sure that the results obtained in this study were not mainly driven by this difference in age, we therefore repeated all the imaging analyses for gray matter (GM) volume and diffusion measures in a subset of sMCI patients $(n=13,75 \pm 6$ years old, 5 female/8 male) matched for age and gender to the pMCI patients.

\section{Imaging parameters}

All patients underwent the same imaging protocol at their first visit, including whole-brain MPRAGE T1-weighted and diffusion- 


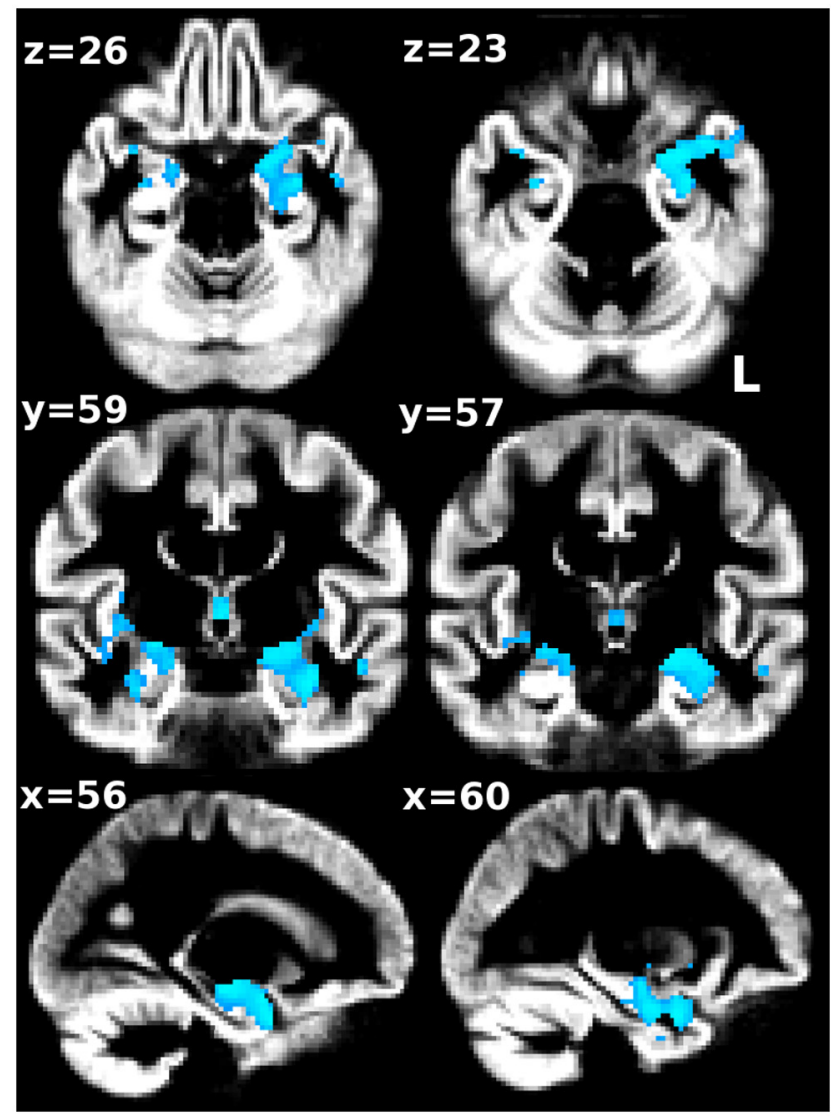

Figure 1. Whole-brain significant $\mathrm{GM}$ volume differences at baseline between $\mathrm{pMCl}$ and SMCI. Despite the $\mathrm{pMCl}$ patients being scanned on average 2.5 years prior to their clinical progression to AD, the FSL-VBM analysis revealed significantly smaller GM volume essentially in the left amygdalo-hippocampal complex compared with SMCI $(p<0.01$ corrected for multiple comparisons). Results are overlaid onto the average of all nonlinearly registered GM segmentations. Radiological convention (left is right).

weighted scans using a 3T Allegra MR imager (Siemens) with a standard quadrature head coil and maximum $40 \mathrm{mT} \cdot \mathrm{m}^{-1}$ gradient capability.

T1-weighted images were obtained with an MPRAGE sequence (TE/ $\left.\mathrm{TI} / \mathrm{TR}=3.5 / 1000 / 2150 \mathrm{~ms}, 1.1 \times 1.1 \times 1.1 \mathrm{~mm}^{3}\right)$. Diffusion-weighted images were obtained using echo-planar imaging (SE-EPI, TE/TR = $89 / 7000 \mathrm{~ms}, 54$ axial slices, bandwidth $=2056 \mathrm{~Hz} /$ voxel, $2.5 \times 2.5 \times 2.5$ $\mathrm{mm}^{3}$ ) with 30 isotropically distributed orientations for the diffusionsensitizing gradients at a $b$ value of $900 \mathrm{~s} \cdot \mathrm{mm}^{-2}$ and $6 b=0$ images. To increase signal-to-noise ratio, scanning was repeated twice and both scans were corrected for head motion and eddy currents using affine registration before being combined.

Direct comparisons between pMCI and sMCI: image processing and statistical analysis

Structural imaging. We carried out an optimized VBM protocol using FMRIB Software Library (FSL) tools (Smith et al., 2004, Douaud et al., 2007; fsl.fmrib.ox.ac.uk/fsl/fslwiki/FSLVBM) to assess the significant differences in the distribution of gray matter volume between pMCI and sMCI. First, a left-right symmetric, study-specific, and unbiased GM template was built from the 13 pMCI patients and from 13 (a random subset) sMCI patients images. Second, the full set of all 35 GM images was nonlinearly spatially normalized onto this study-specific template, "modulated" to compensate for the local contraction/enlargement due to the nonlinear component of the transformation, and smoothed with an isotropic Gaussian kernel with a sigma of $3 \mathrm{~mm}(\sim 7 \mathrm{~mm}$ FWHM) (Good et al., 2001).

Diffusion imaging. Fractional anisotropy (FA) maps-obtained in 12 and $21 \mathrm{MCI}$ subjects, respectively, as diffusion images could not be acquired on one MCI patient for each group (coil failure) —-were generated

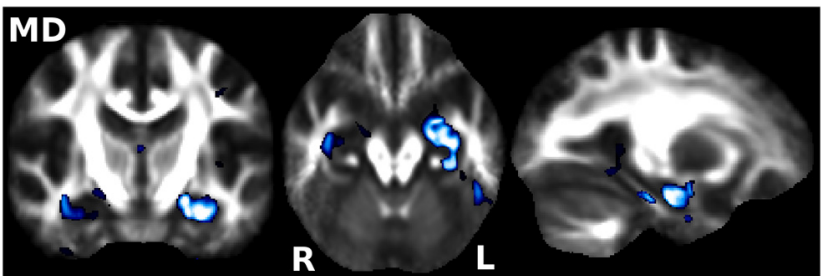

Figure 2. Voxel-based (as opposed to "skeletonized") significant MD differences at baseline. Traditional voxel-based analysis revealed markedly higher MD in $\mathrm{pMCl}$ primarily localized to the left amygdalo-hippocampal complex ( $p<0.05$ corrected for multiple comparisons). Results are overlaid onto the average of all nonlinearly registered FA images. Radiological convention (left is right).
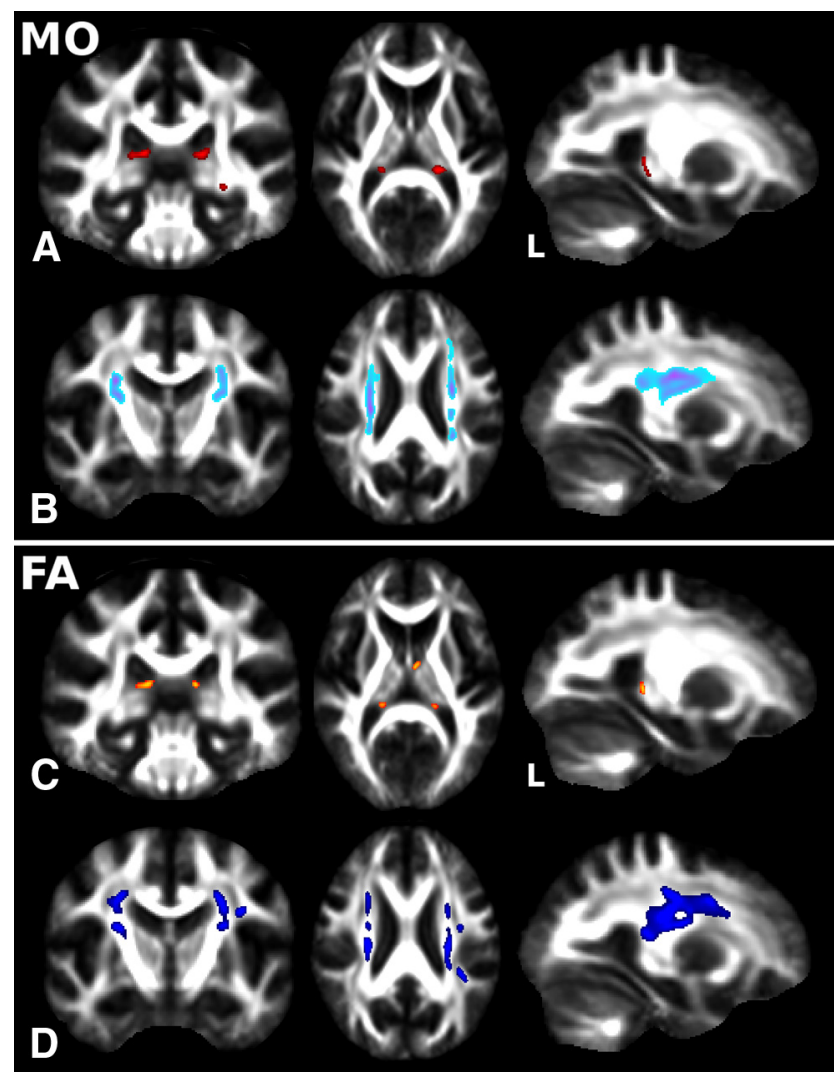

Figure 3. Whole-brain significant WM "skeletonized" $M 0$ and FA differences between $\mathrm{pMC}$ and $S \mathrm{MCl}$ groups at baseline. $\boldsymbol{A}, \boldsymbol{B}$, TBSS revealed significant differences at baseline between $\mathrm{pMCl}$ and $\mathrm{SMCl}$ with lower $\mathrm{M} 0$ in the body of the fornix extending to the left fimbria $(A$, in red) and higher $\mathrm{MO}$ in the centrum semiovale where SLF and CST cross (B, in light blue-purple) $(p<$ 0.05 corrected for multiple comparisons). $C, D, F A$ exhibited the same qualitative changes than MO ( $\boldsymbol{C}$, lower FA in orange; $\boldsymbol{D}$, higher FA in dark blue), although these were just below the statistical threshold ( $p<0.08$ corrected for multiple comparisons). Radiological convention (left is right).

using FSL (Smith et al., 2004). Differences in diffusion tensor imaging (DTI) indices in the white matter were assessed using TBSS, a voxelwise approach that increases the sensitivity and interpretability of the results compared with approaches that are based purely on nonlinear registration (Smith et al., 2006). Because of substantial ventricular enlargement that was seen in many subjects in this study, we created a study-specific FA template by: (1) nonlinearly registering 12 pMCI FA images and 12 (a random subset) sMCI FA images to an FA template in the MNI space derived from 58 healthy subjects (fsl.fmrib.ox.ac.uk/fsl/fslwiki/ FMRIB58_FA); and (2) subsequently averaging those 24 FA images. Similarly to the approach used in FSL-VBM, this study-specific FA template was built with the same number of subjects from each MCI population. 
This was done to avoid any bias that would have consisted in a more accurate nonlinear registration for one of the groups, subsequently making it impossible to distinguish pathologically meaningful differences from registrationrelated differences. Then, we nonlinearly registered the 33 original FA scans to this study-specific FA template. The nearest maximum FA values of each registered FA image were projected onto a white matter skeleton derived from the FMRIB58 template and thresholded at $\mathrm{FA}<0.2$. This projection step aims to remove possible residual effect of cross-subject spatial variability after the nonlinear registration.

We then analyzed with TBSS the mean diffusivity (MD) and mode of anisotropy (MO) images using the resulting warp fields from the FA analysis. We chose to study MO, as it appears to be sensitive in capturing subtle differences in the white matter, in particular in crossing fiber regions, which contrasts with axial and radial diffusivities that prove difficult to interpret in such regions that can encompass up to $90 \%$ of the brain (Wheeler-Kingshott and Cercignani, 2009, Jeurissen et al., 2012). Indeed, in a previous diffusion study looking at the white matter in 61 controls, $56 \mathrm{MCI}$ participants and $53 \mathrm{AD}$ patients (Douaud et al., 2011), no difference could be found when investigating whole-brain diffusion indices of mean diffusivity and fractional anisotropy between MCI and healthy subjects, but we revealed some alteration in a single region of crossing fibers in the centrum semiovale, showing a higher mode of anisotropy (Ennis and Kindlmann, 2006).

Finally, we also investigated differences in GM diffusion-an analysis that is not possible via the TBSS approach, which focuses on white matter (WM) diffusion. The main pitfall of applying a simpler voxelwise approach is that it is not straightforward to robustly account for inaccuracies in the nonlinear registration (with respect to perfectly aligning gray matter across subjects and avoiding partial voluming biases). Bearing this caveat in mind, we looked at voxelwise, as opposed to "skeletonized," FA, $\mathrm{MD}$, and $\mathrm{MO}$ values in the GM. We analyzed the same images processed with TBSS, except that the statistical tests were carried out on the images that had not gone through the final step of projection onto the white matter skeleton.

Statistical analysis. We used permutation-based nonparametric inference within the framework of the general linear model to look for significant abnormalities across the two diagnosis groups (Nichols and Holmes, 2002). Results were considered significant for $p<0.05$, corrected for multiple comparisons after initial cluster-forming thresholding at $p<0.05$ uncorrected. We also looked at correlations between each of the significant MRI-derived measures using datamind, a statistical interface using R developed in BrainVISA (Duchesnay et al., 2004).

\section{Additional analysis: MRI and CSF classification of pMCI} versus $s M C I$

Circular inference concerns prevented us from using the significant differences found when directly comparing $\mathrm{pMCI}$ and sMCI as regions of interest (ROIs) for classification purposes. A common alternative approach (Teipel et al., 2007, Fennema-Notestine et al., 2009, Davatzikos et al., 2011, Oishi et al., 2011) is to use differences found between healthy controls and $\mathrm{AD}$ patients to define ROIs that will be used to extract values in the MCI groups. There is strong evidence, however, that the progression of atrophy from healthy state to AD is a highly spatially and temporally nonlinear process (Ferreira et al., 2011, Tondelli et al., 2012). Therefore, we chose instead to derive the ROIs based on imaging differ-
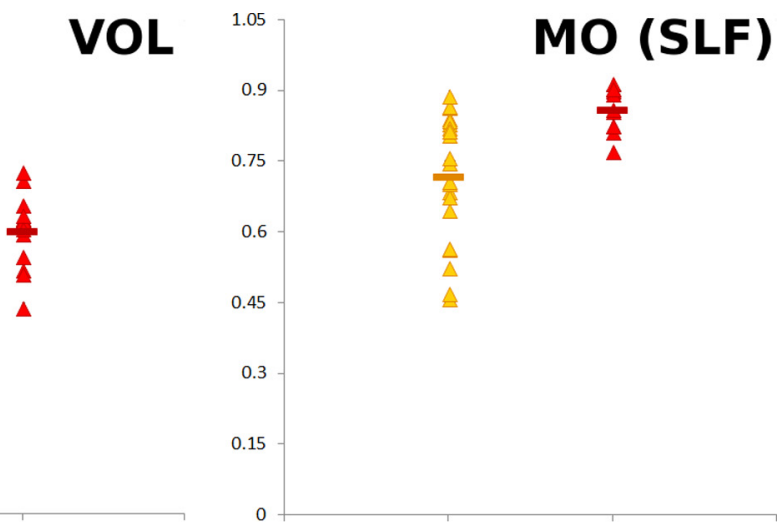

MO (fornix)
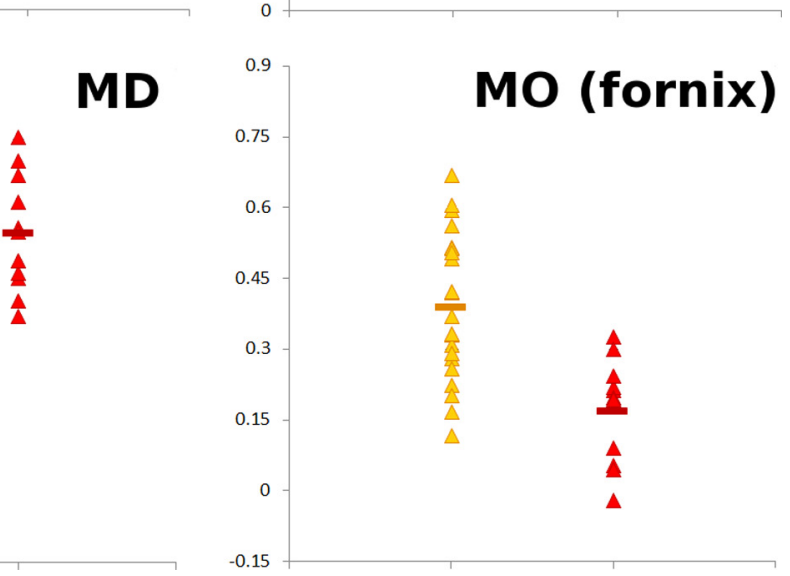

Figure 4. All significant MRI-derived measures in $\mathrm{SMCl}$ (yellow) and $\mathrm{pMCl}$ (red). Values obtained in those regions showing significant differences between the pMCI and sMCI:VOL, GM volume smaller in pMCl essentially in the left hippocampus; MO (SLF), mode of anisotropy higher in pMCl in the SLF; MO (fornix), mode of anisotropy lower in PMCl in the fornix; MD, mean diffusivity higher in pMCl essentially in the left hippocampus $\left(\times 10^{-9} \mathrm{~m}^{2} \cdot \mathrm{s}^{-1}\right)$.

ences found between MCI and AD: our "otherMCI" group and, on the other hand, $59 \mathrm{AD}$ patients without a vascular component. The otherMCI group was composed of the 21 participants who did not meet the "eligibility" criteria for our pMCI and sMCI groups (nine had not yet been clinically followed up for long enough at the time of this study, three were non-amnestic, and nine had progressed to $\mathrm{AD}$ in $<2$ years after scanning). The diagnosis of probable $\mathrm{AD}$ for the 59 patients composing the $\mathrm{AD}$ group was made when both the DSM-IV criteria (American Psychiatric Association, 1994) and the NINCDS-ADRDA criteria (McKhann et al., 1984) were fulfilled (see Douaud et al., 2011 for more details on these patients).

For this classification approach, we carried out the same GM volume and diffusion microstructural images preprocessing as described in the previous section. We then tested the classification accuracy of MRI measures (macrostructural GM volume and diffusion microstructure) and CSF protein concentrations when attempting to classify all 33 amnestic patients into either the pMCI or sMCI group using a linear discriminant analysis and leave-one-out cross-validation in datamind (R).

\section{Results}

Macrostructural differences between pMCI and sMCI at baseline: GM volume

We identified one cluster of significantly smaller GM volume in pMCI compared with sMCI (Fig. 1). This region primarily included the left cornu ammonis of the hippocampus, left laterobasal group of the amygdala, and the left parahippocampal gyrus extending to the temporal pole as identified on the Jülich atlas (Amunts et al., 2005) (fsl.fmrib.ox.ac.uk/fsl/ fslwiki/Atlases). 


\section{Microstructural differences between pMCI and sMCI at baseline: diffusion indices \\ Voxel-based diffusion analysis}

We found significantly higher MD in pMCI compared with sMCI, which was essentially confined to the left hippocampus and amygdala (Fig. 2). We found no significant voxel-based differences in $\mathrm{MO}$ or FA.

\section{TBSS diffusion analysis}

We found two significant regions of difference between pMCI and sMCI using MO. First, we found lower MO in the body of the fornix noticeably extending to the left hippocampus (left fimbria) in pMCI relative to sMCI (Fig. 3A). Second, we found a significantly higher $\mathrm{MO}$ in the centrum semiovale where the superior longitudinal fasciculus (SLF) and the corticospinal tract (CST) cross in pMCI relative to sMCI (Fig. $3 B$ ). We did not find any significant differences in FA or MD on the TBSS white matter skeleton between the two groups after correction for multiple comparisons. However, FA exhibited the same qualitative differences than MO just below threshold for statistical significance (Fig. 3C,D).

As there is considerable enlargement of the ventricles in these patients, we confirmed that the results obtained with TBSS, especially those in the fornix, were interpretable by "deprojecting" all voxels showing significantly higher or lower MO from the white matter skeleton-space back into each subject's full standardspace FA image. This confirmed that the tracts identified on the skeletonized difference maps corresponded well to the fornix and to the region where SLF and CST cross in the centrum semiovale (data not shown).

\section{Macrostructural and microstructural differences separate pMCI and sMCI at baseline}

When looking in the regions defined by the significant differences between pMCI and sMCI in both GM volume (VOL) and diffusion (MD), as well as in WM diffusion (MO), MRI-derived measures proved to differentiate well patients in the pMCI group from those in the sMCI group at baseline (Fig. 4).

When considering the spread of these significant MRI measures, the sMCI patients who had similar imaging values as those of the pMCI patients for their GM volume and MO diffusion ( $x$-axis, Fig. 5) showed nonetheless very distinct MD diffusion values compared to those of pMCI ( $y$-axis, Fig. 5). In all instances, it thus appeared that, following the direction of progression toward $\mathrm{AD}$, what seemed to differentiate the sMCI patients from pMCI was the microstructural diffusion measure of MD in their left hippocampus, above and beyond the traditional macrostructural measure of GM volume reduction of the same structure in particular.

We also found a significantly steeper slope in the correlation between diffusion measures in the hippocampus and in the white matter (MD with MO in the SLF: $p=0.004$; MD with MO in the fornix: $p=0.017$ ) in pMCI compared with sMCI (Fig. 5).

\section{Early prediction of progression to $\mathrm{AD}$}

One important question that we could not answer directly from this dataset (because of the problem of circularity, or "double dipping") was the predictive value of these MRI-derived measures using these regions of significant group differences. We therefore estimated group differences between an MCI group distinct to the pMCI and sMCI groups and a large AD group to obtain the ROIs used to classify our primary groups of interest (sMCI and pMCI). We obtained significantly smaller GM vol-
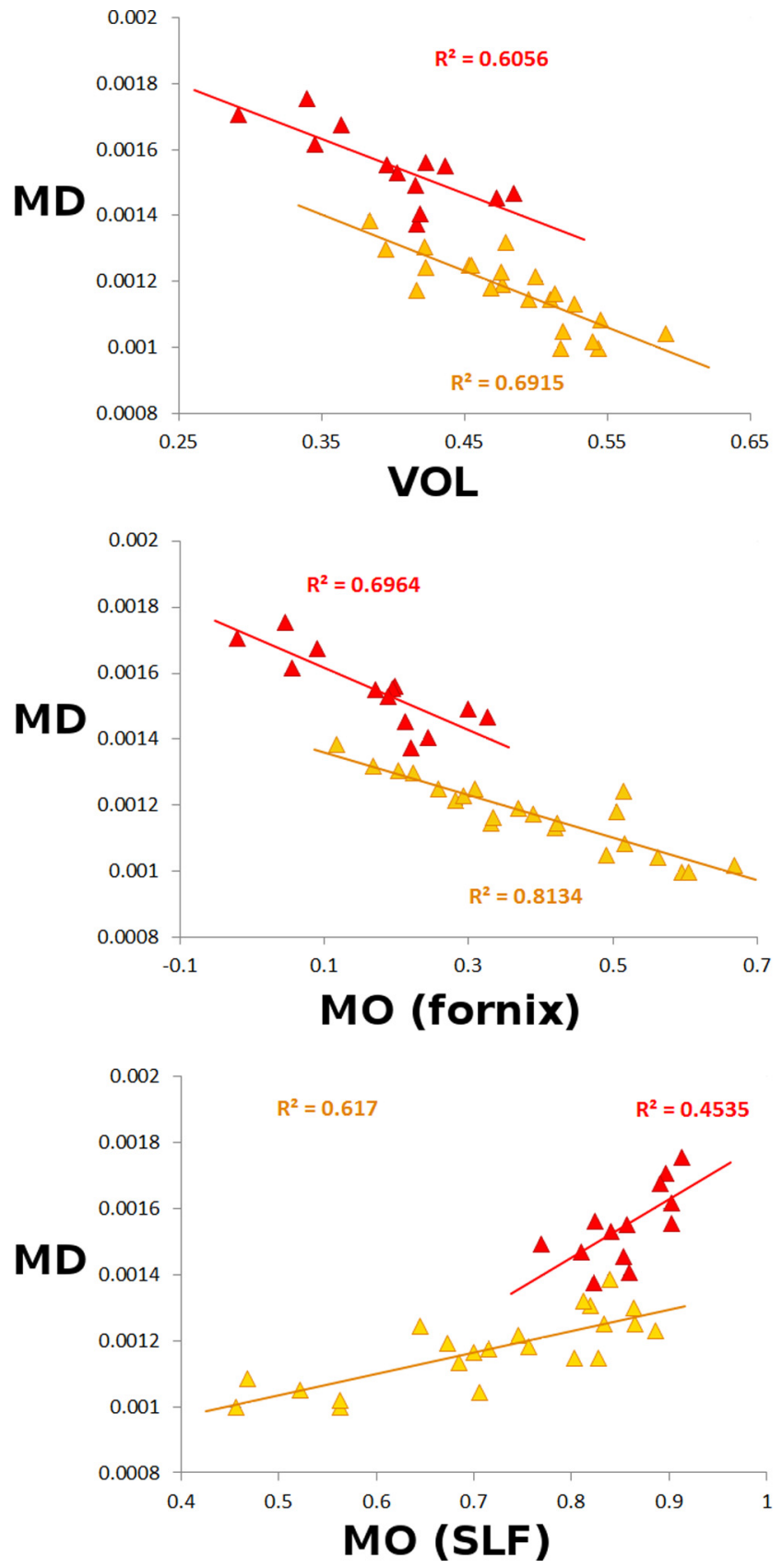

Figure 5. Scatterplots of all significant MRI-derived measures in SMCI (yellow) and PMCI (red). MD values (essentially from the left hippocampus) are plotted against the following: Top, GM volume (VOL) essentially from the left hippocampus; Middle, MO from the fornix; Bottom, MO from the SLF. Top, The relationship between VOL and MD $\left(\times 10^{-6} \mathrm{~m}^{2} \cdot \mathrm{s}^{-1}\right)$ demonstrated a very fine stratification of the two groups with comparable slopes when fitting a linear model for each group considered independently. Middle, The relationship between lower MO and MD showed a slightly steeper slope for the $\mathrm{pMCl}$ group when fitting a linear model for each group independently $(p=0.017)$. Bottom, The relationship between higher M0 and MD clearly showed a steeper slope for the $\mathrm{pMCl}$ group $(p=0.004)$.

ume in the $\mathrm{AD}$ group compared with the MCI group mainly in the left amygdalo-hippocampal complex and bilaterally in the retrolimbic region of the precuneus (data not shown). There was also significantly higher $\mathrm{MO}$ in the centrum semiovale in the $\mathrm{AD}$ group, but no significantly lower MO in the fornix after correction for multiple comparisons. We finally found significantly higher $\mathrm{MD}$ in the $\mathrm{AD}$ group mainly in the left cornu ammonis and laterobasal group, as well as bilaterally in the posterior dentate gyrus (data not shown). 
Using these regions of significant differences as ROIs to extract values from the pMCI and sMCI groups, we found that MD gave the highest classification accuracy of all imaging and CSF measures $(77 \%)$, when we considered each measure as single predictor (Table 3). CSF protein biomarker concentrations had similar or lower discrimination accuracies, with tau concentration providing the best accuracy of the CSF markers (74\%) (Table 3). Finally, by combining imaging and CSF markers, namely by using GM volume $(66 \%$ as single predictor) and a composite of diffusion measures (MO $\times \mathrm{MD}, 69 \%$ as single predictor) together with a composite of CSF measures (tau/A $\beta_{42}, 74 \%$ as single predictor), we could classify accurately 91\% (CI, 75-97) of the amnestic MCI patients with $85 \%$ sensitivity and $96 \%$ specificity and with a diagnostic odds ratio of 116 (CI, 7-4547) (Table 3).
Table 3. Classification accuracy in \% for MRI measures and CSF protein concentration measures

\begin{tabular}{llll}
\hline & Accuracy $(\mathrm{Cl})$ & Sensitivity & Specificity \\
\hline VOL & $66(50-80)$ & 39 & 82 \\
M0 & $66(49-81)$ & 46 & 77 \\
MD & $77(59-89)$ & 62 & 86 \\
VOL $\cup$ MO $\cup$ MD & $69(51-84)$ & 54 & 77 \\
A $\beta_{42}$ & $60(46-75)$ & 31 & 77 \\
Tau & $74(56-88)$ & 39 & 96 \\
p-tau & $66(54-75)$ & 23 & 91 \\
A $\beta_{42} \cup$ tau $\cup$ p-tau & $69(53-81)$ & 39 & 86 \\
V0L/MD & $63(47-78)$ & 39 & 77 \\
M0 $\times$ MD & $69(52-83)$ & 46 & 82 \\
tau/A $\beta_{42}$ & $74(58-84)$ & 46 & 91 \\
$\left(\right.$ VOL/MD or VOL) $\cup$ MO $\times$ MD $\cup$ tau/A $\beta_{42}$ & $91(75-97)$ & 85 & 96 \\
\hline
\end{tabular}

\section{Discussion}

All MRI-derived measures, GM volume, $\mathrm{MD}$, and $\mathrm{MO}$, converged to demonstrate the strong involvement of the left hippocampus in the progression from MCI to $\mathrm{AD}$. We found indeed a markedly smaller GM volume and higher MD in this structure accompanied by lower MO in the body of the fornix extending into the left fimbria. The significantly smaller GM volume in the left hippocampus of pMCI is in line with the recent finding of a VBM meta-analysis on the GM volume predictors of progression to $\mathrm{AD}$ (Ferreira et al., 2011) in which a single cluster was found in the left hippocampus with coordinates corresponding to our main cluster (Fig. 1). Notably, the smaller GM volume was not sufficient to characterize the progression toward $\mathrm{AD}$ (Fig. 5, top): the sMCI patients who had similar left hippocampal volume to the pMCI patients showed nonetheless lower MD values in

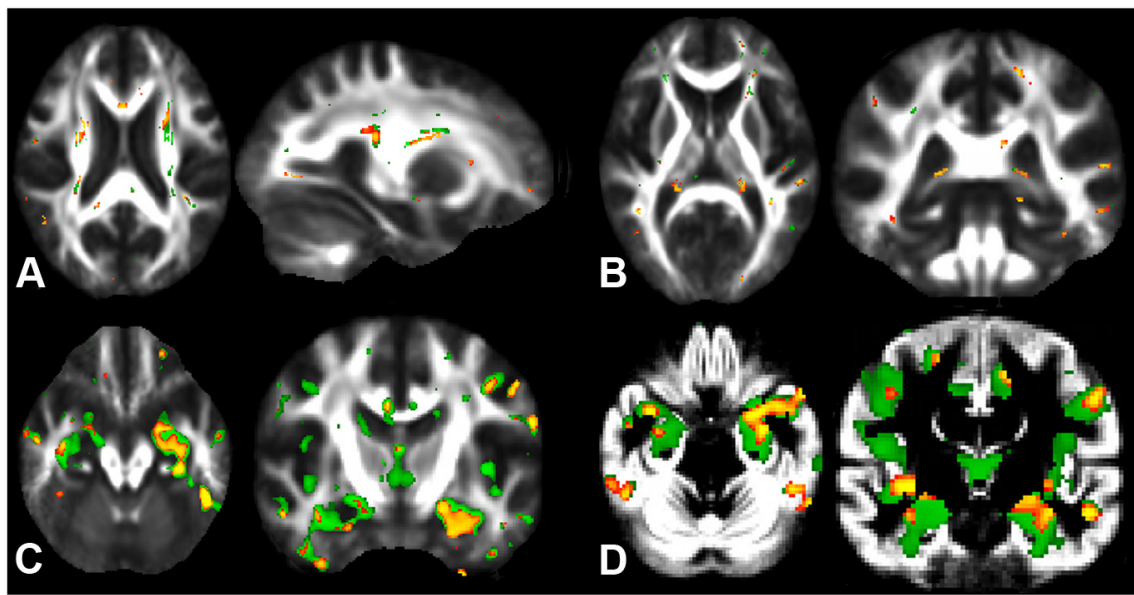

Figure 6. Whole-brain group comparisons between $\mathrm{pMCl}$ and s $\mathrm{MCl}$, after excluding nine sMCl participants from the analyses to match the groups for age and gender. Despite an inherent loss of statistical power in the statistical model due to the loss of these nine subjects, the spatial pattern of the results for each modality was extremely similar between the analyses with the larger dataset (in green) and the reduced, age-matched dataset (in orange). $\boldsymbol{A}$, Higher MO values; $\boldsymbol{B}$, lower MO values; $\boldsymbol{C}$, higher MD values; and $\boldsymbol{D}$, smaller GM volume in $\mathrm{pMCl}$ compared with $\mathrm{SMCI}(p<0.05$ uncorrected, except for $\boldsymbol{C}: p<0.01$, uncorrected). this structure than pMCI patients. Higher

$\mathrm{MD}$ values in the hippocampus of $\mathrm{AD}$ patients compared with healthy controls have previously been shown to remain when controlling for hippocampal volume, demonstrating that diffusion metrics can provide independent information beyond hippocampal volume alone (Salat et al., 2010). In addition, two studies investigating differences between sMCI and pMCI using ROIs in the hippocampus found a trend for higher MD in pMCI (Kantarci et al., 2005, Fellgiebel et al., 2006). If the origin of such higher MD in pMCI cannot be unambiguously elucidated in the present study, it has been related to astrocytosis in a recent combined study of DTI and histology in a model of Huntington's disease (Van Camp et al., 2012). Astrocytosis is a known neuropathological feature of $\mathrm{AD}$ and has been consistently shown to be related to $A \beta$ deposition and neuritic change in the disease (Selkoe, 1991, Cairns et al., 1992, Pike et al., 1994, Steele and Robinson, 2010, Santillo et al., 2011). Speculatively, it is therefore possible that higher MD values, above and beyond the volume reduction in the left hippocampus of $\mathrm{pMCI}$, are a sign of the reactive astroglia typical of $\mathrm{AD}$ stage. The fact that the two MCI groups had similar duration of symptoms (if anything, a slightly shorter duration of symptoms in pMCI, Table 1) argues in favor of a more aggressive neurodegenerative process in pMCI (Ferreira et al., 2011). Of note, a recent study showed that injury to the right hippocampus may be characteristic of a transition from healthy aging to MCI (Tondelli et al., 2012), while we show here that injury to the left hippocampus (as demonstrated by both higher MD and smaller GM volume) may mark the final transition from MCI to AD.

For the first time we found evidence of white matter differences at baseline between pMCI and sMCI groups in the body of the fornix and its left fimbria and in the centrum semiovale SLF (where it crosses the CST). The fornix has been consistently shown in animal studies to be involved in spatial memory formation and visuospatial memory acquisition (Thomas et al., 2011), but the narrowness and location of this tract following the lateral ventricles makes it prone to partial volume effect and very difficult to segment. A few imaging studies have nonetheless demonstrated either a smaller volume or lower FA in this tract in MCI or AD (Copenhaver et al., 2006, Mielke et al., 2009, Zhuang et al., 2010, Agosta et al., 2011). Measures in the SLF, on the other hand, do not suffer from the drawback of partial voluming. This tract, in which lesions result in deficit of visuospatial attention and working memory (Makris et al., 2005, Hoeft et al., 2007), appears in our cohort to be the only white matter pathway stratifying the progression to $\mathrm{AD}$ every step of the way from healthy aging: we previously found that it was showing significant differences be- 
tween healthy, elderly, MCI patients and AD patients (Douaud et al., 2011), and we demonstrate here that it also proves to separate sMCI and pMCI. A recent study has also shown that diffusion differences between non-amnestic and amnestic MCI are found where SLF and CST cross (O'Dwyer et al., 2011). As the GM loss in pMCI compared with sMCI was mostly confined to the medial temporal lobe, the impairment of the SLF is notably the evidence of a subtle degeneration not (yet) detected macroscopically in the corresponding GM regions. Interestingly, however, we found a high correlation between the SLF and all measures associated with the hippocampus (GM volume: $r=-0.69, p<10^{-5}$; MD: $r=0.77, p<10^{-5}$; MO in the fornix and left fimbria: $r=-0.84$, $p<10^{-5}$ ), providing de facto an intermediary between elements of the Papez circuit (hippocampus and fornix) and of the lateral heteromodal cortex (temporoparietal regions, DLPFC) connected via the SLF (Mesulam, 1998). The disruption of the SLF might therefore be a key element that reconciles the apparent discrepancy observed in $\mathrm{AD}$ between the consistent structural alteration seen in the hippocampus and the functional impairment identified in heteromodal association areas (Minoshima et al., 1997, Lustig et al., 2003, Greicius et al., 2004, Buckner et al., 2005, Villain et al., 2008, Buckner et al., 2009, Schroeter et al., 2009).

All three MRI measures showed a very strong difference and limited overlap between pMCI and sMCI. A formal confirmation of the benefit of using MRI measures was that considering alone the best CSF predictors (tau or tau/A $\beta_{42}$ ) led to $74 \%$ accuracy (CI, 56-88), but combining CSF with MRI volume and diffusion measures led to $91 \%$ accuracy (CI, 75 - 97). A qualitatively similar example can be found in one of the largest MCI studies, in which 69 progressing and 170 stable MCI patients were followed up for a maximum of 12 months (Davatzikos et al., 2011). In this setting, combining MRI volumetric measures to the best CSF measure (tau) led to the best classification accuracy (62\%). Additionally, it is reasonable to hope that defining ROIs using a training dataset of pMCI and sMCI (instead of contrasting an entirely different $\mathrm{MCI}$ group with $\mathrm{AD}$ patients as done in this study), or indeed directly using an ROI of the left hippocampus, would lead to an even better prediction accuracy than that achieved here (Desikan et al., 2009).

One limitation (in terms of sensitivity, but not statistical validity) of our study is the limited sample size for each group. We note, though, that the implication of using valid statistical testing (in our case, using permutation-based nonparametric inference fully corrected for multiple comparisons over space) is that a significant result in a small sample is statistically correct, and the actual effect observed has to be larger for a small group to reach significance than it would have needed to be for a larger group (Friston, 2012). Another limitation is that both populations were not matched for age, as we chose instead to focus on a clear preclinical phase of the underlying process leading to $\mathrm{AD}$ and to make sure that both groups were truly different, as the longest period of progression to $\mathrm{AD}$ for the $\mathrm{pMCI}$ group was the shortest period of confirmed clinical stability for the sMCI group. This provided us with 13 and 22 patients in each group who were matched for their proportion of APOE $\varepsilon 4$ carriers, and who did not show any significant differences in duration of symptoms or in their neuropsychological assessment, including episodic memory performance. We therefore additionally reran all analyses in a matched subset of patients $(n=26 \mathrm{in}$ total). Despite the inherent loss of statistical power due to the loss of nine patients, the qualitative pattern of results was extremely similar for each imaging modality to those obtained on the larger dataset ( $n=35$ in total, Fig. 6).
Our findings from structural and diffusion imaging converge to provide evidence of the critical involvement of the left hippocampus and SLF in the progression from $\mathrm{MCI}$ to $\mathrm{AD}$, and this as early as 2.5 years prior to such progression. Direct group comparisons provide MRI-derived differences that separate stable and progressing MCI very well despite both populations showing comparable neuropsychological performance. Best prediction accuracy of $91 \%$ is obtained by combining MRI and CSF measures following the observations made by Jack and others (Vemuri et al., 2009, Jack et al., 2010, Petersen et al., 2010). We also demonstrate that the microstructural measure obtained in the left hippocampus using diffusion imaging shows the most substantial differences between the two groups beyond that of the macrostructural measure of atrophy in the same structure and is the best single predictor of future progression to AD. Diffusion abnormalities in the SLF are also the mark of a subtle microstructural injury not detected by traditional volumetric approach in the GM regions connected by the SLF. To summarize, at the MCI stage, when patients may seek medical advice for the first time, MRI measures of degeneration - and more specifically of microstructural damage — are able to detect the patients who will develop clinical symptoms consistent with probable $\mathrm{AD}$ at least 2 years later. This period of time may offer an opportunity for potential treatments to be effective in slowing the disease progression (Miller, 2012).

\section{References}

Agosta F, Pievani M, Sala S, Geroldi C, Galluzzi S, Frisoni GB, Filippi M (2011) White matter damage in Alzheimer disease and its relationship to gray matter atrophy. Radiology 258:853-863. CrossRef Medline

American Psychiatric Association (1994) Diagnostic and statistical manual of mental disorders: DSM-IV. Washington, DC: American Psychiatric Association.

Amunts K, Kedo O, Kindler M, Pieperhoff P, Mohlberg H, Shah NJ, Habel U, Schneider F, Zilles K (2005) Cytoarchitectonic mapping of the human amygdala, hippocampal region and entorhinal cortex: intersubject variability and probability maps. Anat Embryol (Berl) 210:343-352. CrossRef Medline

Buckner RL, Snyder AZ, Shannon BJ, LaRossa G, Sachs R, Fotenos AF, Sheline YI, Klunk WE, Mathis CA, Morris JC, Mintun MA (2005) Molecular, structural, and functional characterization of Alzheimer's disease: evidence for a relationship between default activity, amyloid, and memory. J Neurosci 25:7709-7717. CrossRef Medline

Buckner RL, Sepulcre J, Talukdar T, Krienen FM, Liu H, Hedden T, AndrewsHanna JR, Sperling RA, Johnson KA (2009) Cortical hubs revealed by intrinsic functional connectivity: mapping, assessment of stability, and relation to Alzheimer's disease. J Neurosci 29:1860-1873. CrossRef Medline

Cairns NJ, Chadwick A, Luthert PJ, Lantos PL (1992) Astrocytosis, beta A4-protein deposition and paired helical filament formation in Alzheimer's disease. J Neurol Sci 112:68-75. CrossRef Medline

Copenhaver BR, Rabin LA, Saykin AJ, Roth RM, Wishart HA, Flashman LA, Santulli RB, McHugh TL, Mamourian AC (2006) The fornix and mammillary bodies in older adults with Alzheimer's disease, mild cognitive impairment, and cognitive complaints: a volumetric MRI study. Psychiatry Res 147:93-103. CrossRef Medline

Costafreda SG, Dinov ID, Tu Z, Shi Y, Liu CY, Kloszewska I, Mecocci P, Soininen H, Tsolaki M, Vellas B, Wahlund LO, Spenger C, Toga AW, Lovestone S, Simmons A (2011) Automated hippocampal shape analysis predicts the onset of dementia in mild cognitive impairment. Neuroimage 56:212-219. CrossRef Medline

Davatzikos C, Bhatt P, Shaw LM, Batmanghelich KN, Trojanowski JQ (2011) Prediction of MCI to AD conversion, via MRI, CSF biomarkers, and pattern classification. Neurobiol Aging 32:2322.e19-2322.e27. CrossRef Medline

Desikan RS, Cabral HJ, Hess CP, Dillon WP, Glastonbury CM, Weiner MW, Schmansky NJ, Greve DN, Salat DH, Buckner RL, Alzheimer's Disease Neuroimaging Initiative (2009) Automated MRI measures identify individuals with mild cognitive impairment and Alzheimer's disease. Brain 132:2048-2057. CrossRef Medline 
Douaud G, Smith S, Jenkinson M, Behrens T, Johansen-Berg H, Vickers J, James S, Voets N, Watkins K, Matthews PM, James A (2007) Anatomically related grey and white matter abnormalities in adolescent-onset schizophrenia. Brain 130:2375-2386. CrossRef Medline

Douaud G, Jbabdi S, Behrens TE, Menke RA, Gass A, Monsch AU, Rao A, Whitcher B, Kindlmann G, Matthews PM, Smith S (2011) DTI measures in crossing-fibre areas: increased diffusion anisotropy reveals early white matter alteration in MCI and mild Alzheimer's disease. Neuroimage 55:880-890. CrossRef Medline

Duchesnay E, Roche A, Riviere D, Papadopoulos D, Cointepas Y, Mangin JE (2004) Population classification based on structural morphometry of cortical sulci. In: Second IEEE international symposium on biomedical imaging: macro to nano, Vol 2, pp 1267-1270. Piscataway Township, NJ: Institute of Electrical and Electronics Engineers. 10.1109/ ISBI.2004.1398776 > <bx $>$

$<$ zrefs\%3B14 > Ennis DB, Kindlmann G $<$ zens $>(2006)<$ zens $>$ Orthogonal tensor invariants and the analysis of diffusion tensor magnetic resonance images. Magn Reson Med 55:136-146. CrossRef Medline

Ewers M, Walsh C, Trojanowski JQ, Shaw LM, Petersen RC, Jack CR Jr, Feldman HH, Bokde AL, Alexander GE, Scheltens P, Vellas B, Dubois B, Weiner M, Hampel H (2012) Prediction of conversion from mild cognitive impairment to Alzheimer's disease dementia based upon biomarkers and neuropsychological test performance. Neurobiol Aging 33:1203-1214. CrossRef Medline

Farrer LA, Cupples LA, Haines JL, Hyman B, Kukull WA, Mayeux R, Myers RH, Pericak-Vance MA, Risch N, van Duijn CM (1997) Effects of age, sex, and ethnicity on the association between apolipoprotein E genotype and Alzheimer disease. A meta-analysis. APOE and Alzheimer Disease Meta Analysis Consortium. JAMA 278:1349-1356. CrossRef Medline

Fellgiebel A, Dellani PR, Greverus D, Scheurich A, Stoeter P, Müller MJ (2006) Predicting conversion to dementia in mild cognitive impairment by volumetric and diffusivity measurements of the hippocampus. Psychiatry Res 146:283-287. CrossRef Medline

Fennema-Notestine C, Hagler DJ Jr, McEvoy LK, Fleisher AS, Wu EH, Karow DS, Dale AM (2009) Structural MRI biomarkers for preclinical and mild Alzheimer's disease. Alzheimer's Disease Neuroimaging Initiative. Hum Brain Mapp 30:3238-3253. CrossRef Medline

Ferreira LK, Diniz BS, Forlenza OV, Busatto GF, Zanetti MV (2011) Neurostructural predictors of Alzheimer's disease: a meta-analysis of VBM studies. Neurobiol Aging 32:1733-1741. CrossRef Medline

Friston K (2012) Ten ironic rules for non-statistical reviewers. Neuroimage 61:1300-1310. CrossRef Medline

Ganguli M (2006) Mild cognitive impairment and the 7 uses of epidemiology. Alzheimer Dis Assoc Disord 20:S52-S57. CrossRef Medline

Gauthier S, Reisberg B, Zaudig M, Petersen RC, Ritchie K, Broich K, Belleville S, Brodaty H, Bennett D, Chertkow H, Cummings JL, de Leon M, Feldman H, Ganguli M, Hampel H, Scheltens P, Tierney MC, Whitehouse P, Winblad B (2006) Mild cognitive impairment. International Psychogeriatric Association Expert Conference. Lancet 367:1262-1270. CrossRef Medline

Good CD, Johnsrude IS, Ashburner J, Henson RN, Friston KJ, Frackowiak RS (2001) A voxel-based morphometric study of ageing in 465 normal adult human brains. Neuroimage 14:21-36. CrossRef Medline

Greicius MD, Srivastava G, Reiss AL, Menon V (2004) Default-mode network activity distinguishes Alzheimer's disease from healthy aging: evidence from functional MRI. Proc Natl Acad Sci U S A 101:4637-4642. CrossRef Medline

Haller S, Nguyen D, Rodriguez C, Emch J, Gold G, Bartsch A, Lovblad KO, Giannakopoulos P (2010) Individual prediction of cognitive decline in mild cognitive impairment using support vector machine-based analysis of diffusion tensor imaging data. J Alzheimers Dis 22:315-327. CrossRef Medline

Hoeft F, Barnea-Goraly N, Haas BW, Golarai G, Ng D, Mills D, Korenberg J, Bellugi U, Galaburda A, Reiss AL (2007) More is not always better: increased fractional anisotropy of superior longitudinal fasciculus associated with poor visuospatial abilities in Williams syndrome. J Neurosci 27:11960-11965. CrossRef Medline

Jack CR Jr, Knopman DS, Jagust WJ, Shaw LM, Aisen PS, Weiner MW, Petersen RC, Trojanowski JQ (2010) Hypothetical model of dynamic biomarkers of the Alzheimer's pathological cascade. Lancet Neurol 9:119-128. CrossRef Medline

Jeurissen B, Leemans A, Tournier JD, Jones DK, Sijbers J (2012) Investigat- ing the prevalence of complex fiber configurations in white matter tissue with diffusion magnetic resonance imaging. Hum Brain Mapp Advance online publication. Retrieved May 19, 2012. doi:10.1002/hbm.22099. CrossRef Medline

Kantarci K, Petersen RC, Boeve BF, Knopman DS, Weigand SD, O’Brien PC, Shiung MM, Smith GE, Ivnik RJ, Tangalos EG, Jack CR Jr (2005) DWI predicts future progression to Alzheimer disease in amnestic mild cognitive impairment. Neurology 64:902-904. CrossRef Medline

Lustig C, Snyder AZ, Bhakta M, O'Brien KC, McAvoy M, Raichle ME, Morris JC, Buckner RL (2003) Functional deactivations: change with age and dementia of the Alzheimer type. Proc Natl Acad Sci U S A 100:14504-14509. CrossRef Medline

Maioli F, Coveri M, Pagni P, Chiandetti C, Marchetti C, Ciarrocchi R, Ruggero C, Nativio V, Onesti A, D’Anastasio C, Pedone V (2007) Conversion of mild cognitive impairment to dementia in elderly subjects: a preliminary study in a memory and cognitive disorder unit. Arch Gerontol Geriatr 44 [Suppl 1]:233-241. CrossRef Medline

Makris N, Kennedy DN, McInerney S, Sorensen AG, Wang R, Caviness VS Jr, Pandya DN (2005) Segmentation of subcomponents within the superior longitudinal fascicle in humans: a quantitative, in vivo, DT-MRI study. Cereb Cortex 15:854-869. CrossRef Medline

McKhann G, Drachman D, Folstein M, Katzman R, Price D, Stadlan EM (1984) Clinical diagnosis of Alzheimer's disease: report of the NINCDS-ADRDA Work Group under the auspices of Department of Health and Human Services Task Force on Alzheimer's Disease. Neurology 34:939-944. CrossRef Medline

Mesulam MM (1998) From sensation to cognition. Brain 121:1013-1052. CrossRef Medline

Mielke MM, Kozauer NA, Chan KC, George M, Toroney J, Zerrate M, Bandeen-Roche K, Wang MC, Vanzijl P, Pekar JJ, Mori S, Lyketsos CG, Albert M (2009) Regionally-specific diffusion tensor imaging in mild cognitive impairment and Alzheimer's disease. Neuroimage 46:47-55. CrossRef Medline

Miller G (2012) Alzheimer's research: stopping Alzheimer's before it starts. Science 337:790-792. CrossRef Medline

Minoshima S, Giordani B, Berent S, Frey KA, Foster NL, Kuhl DE (1997) Metabolic reduction in the posterior cingulate cortex in very early Alzheimer's disease. Ann Neurol 42:85-94. CrossRef Medline

Misra C, Fan Y, Davatzikos C (2009) Baseline and longitudinal patterns of brain atrophy in MCI patients, and their use in prediction of short-term conversion to AD: results from ADNI. Neuroimage 44:1415-1422. CrossRef Medline

Mueller SG, Weiner MW, Thal LJ, Petersen RC, Jack CR, Jagust W, Trojanowski JQ, Toga AW, Beckett L (2005) Ways toward an early diagnosis in Alzheimer's disease: the Alzheimer's Disease Neuroimaging Initiative (ADNI). Alzheimers Dement 1:55-66. CrossRef Medline

Nichols TE, Holmes AP (2002) Nonparametric permutation tests for functional neuroimaging: a primer with examples. Hum Brain Mapp 15:1-25. CrossRef Medline

O’Dwyer L, Lamberton F, Bokde AL, Ewers M, Faluyi YO, Tanner C, Mazoyer B, O'Neill D, Bartley M, Collins DR, Coughlan T, Prvulovic D, Hampel H (2011) Multiple indices of diffusion identifies white matter damage in mild cognitive impairment and Alzheimer's disease. PLoS One 6:e21745. CrossRef Medline

Oishi K, Akhter K, Mielke M, Ceritoglu C, Zhang J, Jiang H, Li X, Younes L, Miller MI, van Zijl PC, Albert M, Lyketsos CG, Mori S (2011) Multimodal MRI analysis with disease-specific spatial filtering: initial testing to predict mild cognitive impairment patients who convert to Alzheimer's disease. Front Neurol 2:54. CrossRef Medline

Petersen RC (2009) Early diagnosis of Alzheimer's disease: is MCI too late? Curr Alzheimer Res 6:324-330. CrossRef Medline

Petersen RC, Aisen PS, Beckett LA, Donohue MC, Gamst AC, Harvey DJ, Jack CR Jr, Jagust WJ, Shaw LM, Toga AW, Trojanowski JQ, Weiner MW (2010) Alzheimer's Disease Neuroimaging Initiative (ADNI): clinical characterization. Neurology 74:201-209. CrossRef Medline

Pike CJ, Cummings BJ, Monzavi R, Cotman CW (1994) Beta-amyloidinduced changes in cultured astrocytes parallel reactive astrocytosis associated with senile plaques in Alzheimer's disease. Neuroscience 63:517-531. CrossRef Medline

Salat DH, Tuch DS, van der Kouwe AJ, Greve DN, Pappu V, Lee SY, Hevelone ND, Zaleta AK, Growdon JH, Corkin S, Fischl B, Rosas HD (2010) 
White matter pathology isolates the hippocampal formation in Alzheimer's disease. Neurobiol Aging 31:244-256. CrossRef Medline

Santillo AF, Gambini JP, Lannfelt L, Långström B, Ulla-Marja L, Kilander L, Engler H (2011) In vivo imaging of astrocytosis in Alzheimer's disease: an (1)(1)C-L-deuteriodeprenyl and PIB PET study. Eur J Nucl Med Mol Imaging 38:2202-2208. CrossRef Medline

Schroeter ML, Stein T, Maslowski N, Neumann J (2009) Neural correlates of Alzheimer's disease and mild cognitive impairment: a systematic and quantitative meta-analysis involving 1351 patients. Neuroimage 47: 1196-1206. CrossRef Medline

Selkoe DJ (1991) The molecular pathology of Alzheimer's disease. Neuron 6:487-498. CrossRef Medline

Smith SM, Jenkinson M, Woolrich MW, Beckmann CF, Behrens TE, Johansen-Berg H, Bannister PR, De Luca M, Drobnjak I, Flitney DE, Niazy RK, Saunders J, Vickers J, Zhang Y, De Stefano N, Brady JM, Matthews PM (2004) Advances in functional and structural MR image analysis and implementation as FSL. Neuroimage [23 Suppl] 1:S208-S219. CrossRef Medline

Smith SM, Jenkinson M, Johansen-Berg H, Rueckert D, Nichols TE, Mackay CE, Watkins KE, Ciccarelli O, Cader MZ, Matthews PM, Behrens TE (2006) Tract-based spatial statistics: voxelwise analysis of multisubject diffusion data. Neuroimage 31:1487-1505. CrossRef Medline

Steele ML, Robinson SR (2010) Reactive astrocytes give neurons less support: implications for Alzheimer's disease. Neurobiol Aging 33: 423.e1-423.e13. CrossRef Medline

Teipel SJ, Born C, Ewers M, Bokde AL, Reiser MF, Möller HJ, Hampel H (2007) Multivariate deformation-based analysis of brain atrophy to predict Alzheimer's disease in mild cognitive impairment. Neuroimage 38: 13-24. CrossRef Medline

Thomas AG, Koumellis P, Dineen RA (2011) The fornix in health and disease: an imaging review. Radiographics 31:1107-1121. CrossRef Medline

Tondelli M, Wilcock GK, Nichelli P, De Jager CA, Jenkinson M, Zamboni G (2012) Structural MRI changes detectable up to ten years before clinical Alzheimer's disease. Neurobiol Aging 33:825.e25-825.e36. CrossRef Medline
Trachtenberg AJ, Filippini N, Mackay CE (2012) The effects of APOEepsilon4 on the BOLD response. Neurobiol Aging 33:323-334. CrossRef Medline

Van Camp N, Blockx I, Camón L, de Vera N, Verhoye M, Veraart J, Van Hecke W, Martínez E, Soria G, Sijbers J, Planas AM, Van der Linden A (2012) A complementary diffusion tensor imaging (DTI)-histological study in a model of Huntington's disease. Neurobiol Aging 33:945-959. CrossRef Medline

Vemuri P, Wiste HJ, Weigand SD, Shaw LM, Trojanowski JQ, Weiner MW, Knopman DS, Petersen RC, Jack CR Jr (2009) MRI and CSF biomarkers in normal, $\mathrm{MCI}$, and $\mathrm{AD}$ subjects: predicting future clinical change. Alzheimer's Disease Neuroimaging Initiative. Neurology 73:294-301. CrossRef Medline

Villain N, Desgranges B, Viader F, de la Sayette V, Mézenge F, Landeau B, Baron JC, Eustache F, Chételat G (2008) Relationships between hippocampal atrophy, white matter disruption, and gray matter hypometabolism in Alzheimer's disease. J Neurosci 28:6174-6181. CrossRef Medline

Wheeler-Kingshott CA, Cercignani M (2009) About "axial" and "radial" diffusivities. Magn Reson Med 61:1255-1260. CrossRef Medline

Whitwell JL, Shiung MM, Przybelski SA, Weigand SD, Knopman DS, Boeve BF, Petersen RC, Jack CR Jr (2008) MRI patterns of atrophy associated with progression to $\mathrm{AD}$ in amnestic mild cognitive impairment. Neurology 70:512-520. CrossRef Medline

Winblad B, Palmer K, Kivipelto M, Jelic V, Fratiglioni L, Wahlund LO, Nordberg A, Bäckman L, Albert M, Almkvist O, Arai H, Basun H, Blennow K, de Leon M, DeCarli C, Erkinjuntti T, Giacobini E, Graff C, Hardy J, Jack C, et al. (2004) Mild cognitive impairment-beyond controversies, towards a consensus: report of the International Working Group on Mild Cognitive Impairment. J Intern Med 256:240-246. CrossRef Medline

Zhuang L, Wen W, Zhu W, Trollor J, Kochan N, Crawford J, Reppermund S, Brodaty H, Sachdev P (2010) White matter integrity in mild cognitive impairment: a tract-based spatial statistics study. Neuroimage 53:16-25. CrossRef Medline 